\title{
Estimation of Heterosis in the Advanced Lines of Bottle Gourd [Lagenaria siceraria (Mol.) Standl.] for Growth, Earliness and Yield Parameters
}

\author{
Raju K. Khot ${ }^{1 *}$, Shashikanth Evoor ${ }^{2}$, Vilas D. Gasti ${ }^{2}$, Sumangala Koulagi ${ }^{3}$ and \\ Dileepkumar A. Masuthi ${ }^{4}$
}

\author{
${ }^{1}$ Department of Vegetable Science, ${ }^{3}$ Department of Plant Pathology, \\ ${ }^{4}$ Department of Seed Science and Technology, K. R. C. College of Horticulture, Arabhavi, Tq- \\ Gokak- 591222, Karnataka, India \\ ${ }^{2}$ Department of Vegetable Science, College of Horticulture, Bagalkot-587102, \\ Karnataka, India
}

*Corresponding author

\begin{tabular}{|l|l|}
\hline \multicolumn{1}{c|}{ A B S T R A C T } \\
\cline { 2 - 3 } \multicolumn{1}{l|}{ Key words } & $\begin{array}{l}\text { The present investigation was carried out with the objective to assess the magnitude and } \\
\text { direction of heterosis and to identify good combiners for growth, earliness and yield } \\
\text { characters. The maximum heterosis over better parent, the best parent and the commercial } \\
\text { check was observed in the cross Bot.G-6-2 X Arka Bahar for all the growth parameters } \\
\text { studied except heterobeltiosis for vine length. The cross Bot.G-6-2 X Pusa Nagenaria } \\
\text { siceraria (Mol.) Standl.], } \\
\text { Vegetable }\end{array}$ \\
\hline Article Info & $\begin{array}{l}\text { exhibited maximum and significant heterobeltiosis for vine length. The crosses Bot.G-2 X } \\
\text { Arka Bahar, Bot.G-6-1 X Samrat, Kolkotta Collection X Arka Bahar and Kolkotta } \\
\text { Collection X Samrat were found promising for earliness. The crosses Bot.G-6-1 X Samrat } \\
\text { and Kolkotta Collection X Samrat (22.93\%) followed by Bot.G-6 X Samrat (21.05\%) } \\
\text { showed significant heterosis over the commercial check in order of merit for fruit yield per } \\
\text { vine. }\end{array}$ \\
\hline $\begin{array}{l}\text { Accepted: } \\
\text { Available Online: } \\
\text { 10 September 2018 }\end{array}$
\end{tabular}

\section{Introduction}

Bottle gourd [Lagenaria siceraria (Mol.) Standl.] is one of the important cucurbitaceous vegetable having wide range of uses and it is cultivated largely in the tropics and subtropics for its edible fruits. It is commonly called as calabash gourd, trumpet gourd, white flowered gourd and zucca melon. Bottle gourd is a commonly grown vegetable in India having chromosome number of $2 \mathrm{n}=22$. Heterosis signifies increased or decreased vigour of the $F_{1}$ hybrids over the parents. Some bottle gourd hybrids Pusa Meghdoot and Pusa Manjari were released, long back in 1971, by Indian Agricultural Research Institute, New Delhi. The commercial exploitation of hybrid vigour depends on the ease with which the technique employed for hybrid seed production and reasonably the low cost of $F_{1}$ seed production. 
The commercial usefulness of the hybrid would depend on its performance in comparison to the best existing commercial hybrid. Hence, the present investigation was undertaken to study the magnitude and direction of heterosis for growth, earliness and yield parameters.

\section{Materials and Methods}

The investigation on "Estimation of heterosis in advanced lines of bottle gourd [Lagenaria siceraria (mol.) standl.] for growth, earliness and yield parameters" was undertaken at the Department of Vegetable Science, K. R. C. College of Horticulture, Arabhavi, Gokak Taluk, Belagavi district of Karnataka state. The experimental material comprised of 10 lines which were collected from the department itself and 3 testers (Arka Bahar, Pusa Naveen and Samrat) collected from different sources and their $30 \mathrm{~F}_{1}$ hybrids along with one commercial check (Warad). Each of the 10 lines was crossed with each of the 3 testers to derive $30 \mathrm{~F}_{1}$ hybrids in line $\mathrm{x}$ tester fashion. The experiment was laid out in randomized block design with two replications. Ridges and furrows were opened at a distance of three meters apart. Two to three seeds of each genotype per hill were dibbled at a distance of $90 \mathrm{~cm}$ in a row. Five randomly choosen plants in each replication of all the entries were labelled and used for recording the observations. The mean of five plants was taken for statistical analysis.

\section{Results and Discussion}

The analysis of variance revealed that significant differences among treatments for all the traits indicating the presence of appreciable genetic diversity among the parents and cross combinations. Heterosis for growth parameters is an indication of heterosis for yield as growth and yield parameters are strongly associated. Significant and high magnitude of heterosis over better parent, the best parent and the commercial check was observed in the desirable direction for all the growth, earliness and yield parameters. Heterosis for growth and earliness parameters are presented in Table 1 to 5 .

Out of 30 crosses, two crosses over better parent, ten crosses over the best parent and one cross over the commercial check exhibited positive and significant heterosis for vine length. The maximum positive heterosis over better parent was observed in the cross Bot.G6-2 X Pusa Naveen (27.37\%), whereas the cross Bot.G-6-2 X Arka Bahar exhibited maximum heterosis over the best parent $(80.68 \%)$ and over the commercial check (38.67\%). Heterosis over better parent and the commercial check was also reported by several workers on bottle gourd for vine length (Dubey and Maurya, 2003; Sharma et al., 2012; Singh et al., 2012; Yadav and Kumar, 2012; Ray et al., 2015; Adarsh et al., 2017).

For number of leaves two crosses over better parent, one cross over the best parent and five crosses over the commercial check exhibited positive and significant heterosis. The maximum heterosis over better parent $(86.31 \%)$, the best parent $(34.70 \%)$ and the commercial check $(92.32 \%)$ was observed in the cross Bot.G-6-2 X Arka Bahar. Heterosis over better parent, the best parent and the commercial check was also reported by Gayakwad (2014).

Out of 30 crosses, seven crosses over better parent, one cross over the best parent and nine crosses over the commercial check exhibited positive and significant heterosis for number of branches. The cross Bot.G-6-2 X Arka Bahar showed the highest heterosis over better parent (57.14\%), over the best parent $(10.00 \%)$ and over the commercial check (76.00\%). Heterosis over better parent, the 
best parent and the commercial check was also reported by Sharma et al., (2012), Singh et al., (2012), Ray et al., (2015) and Ghuge et al., (2016).

Earliness indicated by negative estimates of heterosis is well recognized and prime objective of any hybrid breeding programme. Days to first male and female flowering, number of nodes to female flower and days to first harvest are the indicating traits for early yield. For all these characters, significant variability was observed among the genotypes and significant negative heterosis was observed to be desirable.

For days to first male flowering, out of 30 crosses, four crosses over better parent, one cross over the best parent and 12 crosses over the commercial check exhibited negative and significant heterosis.

The maximum and significant negative heterosis over better parent $(-3.96 \%)$ was observed in the cross Bot.G-6-1 X Pusa Naveen and over the best parent $(-3.13 \%)$ and over the commercial check $(-7.92 \%)$ was observed in the cross Bot.G-2 X Arka Bahar. Similar frequency of heterobeltosis and standard heterosis for days to first male flowering was also reported by Yadav and Kumar (2012) and Gayakawad (2014) in bottle gourd.

For days to first female flowering, among 30 hybrids, two hybrids exhibited significant negative heterosis over better parent and five hybrids exhibited negative significant heterosis over the commercial check. The maximum and significant negative heterosis over better parent $(-5.50 \%)$ was observed in the cross Bot.G-2-1 X Pusa Naveen and the cross Bot.G-6-1 X Samrat exhibited the significant and negative heterosis over commercial check (-6.42\%). Heterosis with reference to days to female flower appearance was also reported by Pandey et al., (2004), Yadav and Kumar (2012) and Adarsh et al., (2017) in bottle gourd.

Nodes to first female flower are considered as index of earliness. For number of nodes at first female flower, one crosses over better parent and six crosses over commercial check in negative (desirable) and significant heterosis. The cross Bot.G-6 X Samrat exhibited significant and negative heterosis over better parent $(-6.16 \%)$ and over commercial check ($18.45 \%)$. Similarly significant and high magnitude of heterosis over the better parent and negative heterosis over commercial check in desirable direction was also reported by Pandey et al., (2004) in bottle gourd.

For days to first harvest, out of 30 crosses, one cross over better parent and three crosses over the commercial check exhibited significant negative heterosis in the desirable (negative) direction, the cross Bot.G-6-1 X Samrat exhibited significant and negative heterosis over better parent $(-5.04 \%)$ and the cross Kolkotta Collection X Pusa Naveen over commercial check $(-7.14 \%)$. Significant and negative heterosis over the better parent $(-1.93$ to $-5.35 \%$ ) was also reported by Ojha et al., (2009) and over commercial check (-1.05 to $6.74 \%$ ) was also reported by Ghuge et al., (2016) in bottle gourd.

For days to last harvest, among 30 crosses, two crosses over better parent and four crosses over the commercial check exhibited significant positive heterosis.

The significant positive heterosis was observed in the cross Bot.G-7-1 X Samrat over better parent (12.08\%) and over commercial check $(7.37 \%)$ was observed in the cross Bot.G-4-1 X Samrat. This is little high as compared to earlier reports of Naliyadhara et al., (2007) over better parent $(2.70 \%)$ and over commercial check $(4.87 \%)$. 
Table.1 Heterosis (\%) over better parent, the best parent and the commercial check for vine length and number of leaves in bottle gourd

\begin{tabular}{|c|c|c|c|c|c|c|c|}
\hline \multirow{2}{*}{$\begin{array}{c}\text { Sl. } \\
\text { No. }\end{array}$} & \multirow[t]{2}{*}{ Cross } & \multicolumn{3}{|c|}{ Vine length } & \multicolumn{3}{|c|}{ Number of leaves } \\
\hline & & BP & BTP & $\mathrm{CC}$ & BP & BTP & $\mathrm{CC}$ \\
\hline 1 & Bot.G-2 X AB & - & $-45.06 * *$ & - & - & - & - \\
\hline 2 & Bot.G-2 X PN & $-8.97 * *$ & $-6.72 * *$ & - & -0.99 & - & -4.33 \\
\hline 3 & Bot.G-2 X S & - & $6.89 * *$ & - & - & - & -1.08 \\
\hline 4 & Bot.G-2-1 X AB & - & $16.68^{* *}$ & - & - & - & - \\
\hline 5 & Bot.G-2-1 X PN & - & $-2.81 * *$ & - & - & - & $15.37 * *$ \\
\hline 6 & Bot.G-2-1 X S & - & $-37.53 * *$ & - & - & - & - \\
\hline 7 & Bot.G-4 X AB & - & $-25.45 * *$ & - & - & - & - \\
\hline 8 & Bot.G-4 X PN & - & $-22.20 * *$ & - & - & - & - \\
\hline 9 & Bot.G-4 X S & - & $-15.66^{* *}$ & - & - & - & - \\
\hline 10 & Bot.G-4-1 X AB & - & $-28.00 * *$ & - & - & - & - \\
\hline 11 & Bot.G-4-1 X PN & - & $-16.38^{* *}$ & - & - & - & - \\
\hline 12 & Bot.G-4-1 X S & - & $18.30^{* *}$ & $-9.21 * *$ & -4.41 & - & $12.66 * *$ \\
\hline 13 & Bot.G-6 X AB & - & $25.36^{* *}$ & -3.79 & -3.24 & - & $16.45 * *$ \\
\hline 14 & Bot.G-6 X PN & - & $-34.38 * *$ & - & - & - & - \\
\hline 15 & Bot.G-6 X S & - & $-19.62 * *$ & - & - & - & - \\
\hline 16 & Bot.G-6-1 X AB & - & $15.66^{* *}$ & - & - & - & - \\
\hline 17 & Bot.G-6-1 X PN & - & $-29.11 * *$ & - & - & - & - \\
\hline 18 & Bot.G-6-1 X S & - & -2.55 & - & - & - & -4.76 \\
\hline 19 & Bot.G-6-2 X AB & $25.18 * *$ & $80.68^{* *}$ & $38.67 * *$ & $86.31 * *$ & $34.70 * *$ & $92.32 * *$ \\
\hline 20 & Bot.G-6-2 X PN & $27.37 * *$ & $16.43^{* *}$ & - & $30.83^{* *}$ & - & $7.27 * *$ \\
\hline 21 & Bot.G-6-2 X S & - & $6.47 * *$ & - & - & - & 1.15 \\
\hline 22 & Bot.G-7 X AB & - & $-14.55^{* *}$ & - & - & - & - \\
\hline 23 & Bot.G-7 X PN & - & $-42.51 * *$ & - & - & - & - \\
\hline 24 & Bot.G-7 X S & - & $-35.87 * *$ & - & - & - & - \\
\hline 25 & Bot.G-7-1 X AB & - & $-28.85 * *$ & - & - & - & - \\
\hline 26 & Bot.G-7-1 X PN & $-7.28 * *$ & $-9.70 * *$ & - & - & - & - \\
\hline 27 & Bot.G-7-1 X S & - & -0.21 & - & - & - & - \\
\hline 28 & KC X AB & - & $-27.45^{* *}$ & - & - & - & - \\
\hline 29 & KC X PN & $-8.85^{* *}$ & $26.30^{* *}$ & -3.07 & - & - & $-9.91 * *$ \\
\hline 30 & KC X S & - & $23.17 * *$ & $-5.47 *$ & - & - & 2.23 \\
\hline & S.Em \pm & 1.37 & 1.37 & 1.37 & 1.95 & 1.95 & 1.95 \\
\hline & C.D. @ $5 \%$ & 3.91 & 3.91 & 3.91 & 5.56 & 5.56 & 5.56 \\
\hline & C.D. @ 1\% & 5.22 & 5.22 & 5.22 & 7.44 & 7.44 & 7.44 \\
\hline
\end{tabular}

*and ** indicate significance of values at $\mathrm{p}=0.05$ and 0.01 , respectively. BP- Heterosis over better parent BTPHeterosis over the best parent, CC- Heterosis over the commercial check, DAS- Days after sowing, KC-Kolkotta collection, AB- Arka Bahar, PN- Pusa Naveen, S-Samrat 
Table.2 Heterosis (\%) over better parent, the best parent and the commercial check for number of branches and days to first flowering in bottle gourd

\begin{tabular}{|c|c|c|c|c|c|c|c|}
\hline SI. & \multirow[t]{2}{*}{ Cross } & \multicolumn{3}{|c|}{ Number of branches } & \multicolumn{3}{|c|}{ Days to first flowering } \\
\hline No & & BP & BTP & $\mathrm{CC}$ & BP & BTP & $\mathrm{CC}$ \\
\hline 1 & Bot.G-2 X AB & - & $-62.50 * *$ & - & $-3.13 * *$ & $-3.13 * *$ & $-7.92 * *$ \\
\hline 2 & Bot.G-2 X PN & $-2.08 * *$ & $-41.25 * *$ & $-6.00 * *$ & -1.01 & $2.08 *$ & $-2.97 * *$ \\
\hline 3 & Bot.G-2 X S & - & $-40.63 * *$ & $-5.00 * *$ & $4.12 * *$ & $5.21 * *$ & 0.00 \\
\hline 4 & Bot.G-2-1 X AB & - & $-50.00 * *$ & - & $7.29 * *$ & $7.29 * *$ & $1.98 *$ \\
\hline 5 & Bot.G-2-1 X PN & - & $-46.25 * *$ & - & -0.99 & $4.17 * *$ & -0.99 \\
\hline 6 & Bot.G-2-1 X S & - & $-56.25 * *$ & - & $2.06 *$ & $3.13 * *$ & $-1.98 *$ \\
\hline 7 & Bot.G-4 X AB & - & $-57.50 * *$ & - & $5.21 * *$ & $5.21 * *$ & 0.00 \\
\hline 8 & Bot.G-4 X PN & - & $-55.63 * *$ & - & -1.00 & $3.13 * *$ & $-1.98 *$ \\
\hline 9 & Bot.G-4 X S & - & $-50.00 * *$ & - & $2.06^{*}$ & $3.13 * *$ & $-1.98 *$ \\
\hline 10 & Bot.G-4-1 X AB & - & $-60.25 * *$ & - & $3.13 * *$ & $3.13 * *$ & $-1.98 *$ \\
\hline 11 & Bot.G-4-1 X PN & - & $-52.50 * *$ & - & 1.01 & $4.17 * *$ & -0.99 \\
\hline 12 & Bot.G-4-1 X S & 0.00 & 0.00 & $60.00 * *$ & $4.12 * *$ & $5.21 * *$ & 0.00 \\
\hline 13 & Bot.G-6 X AB & $10.00 * *$ & $-17.50 * *$ & $32.00 * *$ & $4.17 * *$ & $4.17 * *$ & -0.99 \\
\hline 14 & Bot.G-6 X PN & - & $-57.50 * *$ & - & -0.99 & $4.17 * *$ & -0.99 \\
\hline 15 & Bot.G-6 X S & - & $-52.50 * *$ & - & $6.19 * *$ & $7.29 * *$ & $1.98 *$ \\
\hline 16 & Bot.G-6-1 X AB & - & $-42.50 * *$ & $-8.00 * *$ & $5.21 * *$ & $5.21 * *$ & 0.00 \\
\hline 17 & Bot.G-6-1 X PN & - & $-57.50 * *$ & - & $-3.96 * *$ & 1.04 & $-3.96 * *$ \\
\hline 18 & Bot.G-6-1 X S & $-1.69 * *$ & $-27.50 * *$ & $16.00 * *$ & $4.12 * *$ & $5.21 * *$ & 0.00 \\
\hline 19 & Bot.G-6-2 X AB & $57.14 * *$ & $10.00 * *$ & $76.00 * *$ & $3.13 * *$ & $3.13 * *$ & $-1.98 *$ \\
\hline 20 & Bot.G-6-2 X PN & $54.55^{* *}$ & $-15.00 * *$ & $36.00 * *$ & $2.00 *$ & $6.25 * *$ & 0.99 \\
\hline 21 & Bot.G-6-2 X S & - & $-35.00 * *$ & $4.00 * *$ & $-2.06 *$ & -1.04 & $-5.94 * *$ \\
\hline 22 & Bot.G-7 X AB & - & $-47.50 * *$ & - & $9.38 * *$ & $9.38 * *$ & $3.96 * *$ \\
\hline 23 & Bot.G-7 X PN & 0.00 & $-52.50 * *$ & - & $-1.98^{*}$ & $3.13 * *$ & $-1.98 *$ \\
\hline 24 & Bot.G-7 X S & - & $-55.00 * *$ & - & $8.25 * *$ & $9.38 * *$ & $3.96 * *$ \\
\hline 25 & Bot.G-7-1 X AB & - & $-65.00 * *$ & - & $9.38 * *$ & $9.38 * *$ & $3.96 * *$ \\
\hline 26 & Bot.G-7-1 X PN & $25.00 * *$ & $-37.50 * *$ & 0.00 & 1.01 & $4.17 * *$ & -0.99 \\
\hline 27 & Bot.G-7-1 X S & $18.64 * *$ & $-12.50 * *$ & $40.00 * *$ & $6.19 * *$ & $7.29 * *$ & $1.98^{*}$ \\
\hline 28 & KC X AB & - & $-62.50 * *$ & - & -1.04 & -1.04 & $-5.94 * *$ \\
\hline 29 & KC X PN & $12.77 * *$ & $-33.75^{* *}$ & $6.00 * *$ & $4.12 * *$ & $5.21 * *$ & 0.00 \\
\hline 30 & KC X S & $1.69 * *$ & $-25.00 * *$ & $20.00 * *$ & 1.03 & $2.08 *$ & $-2.97 * *$ \\
\hline & S.Em \pm & 0.20 & 0.20 & 0.20 & 0.59 & 0.59 & 0.59 \\
\hline & C.D. @ $5 \%$ & 0.57 & 0.57 & 0.57 & 1.69 & 1.69 & 1.69 \\
\hline & C.D.@1\% & 0.77 & 0.77 & 0.77 & 2.25 & 2.25 & 2.25 \\
\hline
\end{tabular}

*and ** indicate significance of values at $\mathrm{p}=0.05$ and 0.01 , respectively. BP- Heterosis over better parent BTPHeterosis over the best parent CC- Heterosis over the commercial check, DAS- Days after sowing, KC-Kolkotta collection, $\mathrm{AB}=$ Arka Bahar, $\mathrm{PN}=$ Pusa Naveen, S-Samrat 
Table.3 Heterosis (\%) over better parent, the best parent and the commercial check for days to first female flower and nodes upto first female flower in bottle gourd

\begin{tabular}{|c|c|c|c|c|c|c|c|}
\hline \multirow{2}{*}{$\begin{array}{l}\text { Sl. } \\
\text { No } \\
\end{array}$} & \multirow[t]{2}{*}{ Cross } & \multicolumn{3}{|c|}{ Days to first female flower } & \multicolumn{3}{|c|}{ Nodes upto first female } \\
\hline & & RP & RTP & C.C. & RP & RTP & C.C. \\
\hline 1 & Bot.G-2 X AB & $12.75 * *$ & $26.37 * *$ & $5.50 * *$ & $16.27 * *$ & $55.71 * *$ & $3.81 * *$ \\
\hline 2 & Bot.G-2 X PN & $5.50 * *$ & $26.37 * *$ & $5.50 * *$ & $23.33 * *$ & $65.18 * *$ & $10.12 * *$ \\
\hline 3 & Bot.G-2 X S & $18.68 * *$ & $18.68 * *$ & -0.92 & $8.22 * *$ & $41.07 * *$ & $-5.95 * *$ \\
\hline 4 & Bot.G-2-1 X AB & $11.76 * *$ & $25.28 * *$ & $4.59 * *$ & $6.06 * *$ & $56.25 * *$ & $4.17 * *$ \\
\hline 5 & Bot.G-2-1 X PN & $-5.50 * *$ & $13.19 * *$ & $-5.50 * *$ & $15.63 * *$ & $65.18 * *$ & $10.12 * *$ \\
\hline 6 & Bot.G-2-1 X S & $19.78 * *$ & $19.78 * *$ & 0.00 & $13.01 * *$ & $47.32 * *$ & $-1.79 * *$ \\
\hline 7 & Bot.G-4 X AB & $6.86^{* *}$ & $19.78 * *$ & 0.00 & $12.62 * *$ & $55.36 * *$ & $3.57 * *$ \\
\hline 8 & Bot.G-4 X PN & 1.87 & $19.78 * *$ & 0.00 & $21.68 * *$ & $67.86 * *$ & $11.90 * *$ \\
\hline 9 & Bot.G-4 X S & $24.18 * *$ & $24.18 * *$ & $3.67 * *$ & $36.99 * *$ & $78.57 * *$ & $19.05 * *$ \\
\hline 10 & Bot.G-4-1 X AB & $10.78 * *$ & $24.18 * *$ & $3.67 * *$ & $15.00 * *$ & $64.29 * *$ & $9.52 * *$ \\
\hline 11 & Bot.G-4-1 X PN & 0.00 & $18.68 * *$ & -0.92 & $1.25^{*}$ & $44.64 * *$ & $-3.57 * *$ \\
\hline 12 & Bot.G-4-1 X S & $26.37 * *$ & $26.37 * *$ & $5.50 * *$ & $15.75 * *$ & $50.89 * *$ & 0.60 \\
\hline 13 & Bot.G-6 X AB & $9.80 * *$ & $23.08 * *$ & $2.75^{* *}$ & $18.39 * *$ & $83.93 * *$ & $22.62 * *$ \\
\hline 14 & Bot.G-6 X PN & $3.67 * *$ & $24.18 * *$ & $3.67 * *$ & $11.25 * *$ & $58.93 * *$ & $5.95 * *$ \\
\hline 15 & Bot.G-6 X S & $27.47 * *$ & $27.47 * *$ & $6.42 * *$ & $-6.16 * *$ & $22.32 * *$ & $-18.45 * *$ \\
\hline 16 & Bot.G-6-1 X AB & $10.78 * *$ & $24.18 * *$ & $3.67 * *$ & $6.90 * *$ & $66.07 * *$ & $10.71 * *$ \\
\hline 17 & Bot.G-6-1 X PN & $3.67 * *$ & $24.18 * *$ & $3.67 * *$ & $13.75^{* *}$ & $62.50 * *$ & $8.33 * *$ \\
\hline 18 & Bot.G-6-1 X S & $12.09 * *$ & $12.09 * *$ & $-6.42 * *$ & $27.40 * *$ & $66.07 * *$ & $10.71 * *$ \\
\hline 19 & Bot.G-6-2 X AB & $9.80 * *$ & $23.08 * *$ & $2.75^{* *}$ & $30.77 * *$ & $51.79 * *$ & $1.19 *$ \\
\hline 20 & Bot.G-6-2 X PN & $2.75^{* *}$ & $23.08 * *$ & $2.75^{* *}$ & $29.23 * *$ & $50.00 * *$ & 0.00 \\
\hline 21 & Bot.G-6-2 X S & $23.08 * *$ & $23.08 * *$ & $2.75^{* *}$ & $40.00 * *$ & $62.50 * *$ & $8.33 * *$ \\
\hline 22 & Bot.G-7 X AB & $8.82 * *$ & $21.98 * *$ & 1.83 & $46.43 * *$ & $46.43 * *$ & $-2.38 * *$ \\
\hline 23 & Bot.G-7 X PN & $2.75 * *$ & $23.08 * *$ & $2.75^{* *}$ & $73.21 * *$ & $73.21 * *$ & $15.48 * *$ \\
\hline 24 & Bot.G-7 X S & $20.88 * *$ & $20.88 * *$ & 0.92 & $41.07 * *$ & $41.07 * *$ & $-5.95 * *$ \\
\hline 25 & Bot.G-7-1 X AB & $14.71 * *$ & $28.57 * *$ & $7.34 * *$ & $11.98 * *$ & $66.96 * *$ & $11.31 * *$ \\
\hline 26 & Bot.G-7-1 X PN & 1.83 & $21.98 * *$ & 1.83 & $18.75^{* *}$ & $69.64 * *$ & $13.10 * *$ \\
\hline 27 & Bot.G-7-1 X S & $28.57 * *$ & $28.57 * *$ & $7.34 * *$ & $20.55^{* *}$ & $57.14 * *$ & $4.76 * *$ \\
\hline 28 & KC X AB & $3.92 * *$ & $16.48 * *$ & $-2.75 * *$ & $8.05 * *$ & $67.86 * *$ & $11.90 * *$ \\
\hline 29 & KC X PN & $-2.80 * *$ & $14.29 * *$ & $-4.59 * *$ & $12.50 * *$ & $60.71 * *$ & $7.14 * *$ \\
\hline 30 & KC X S & $15.38 * *$ & $15.39 * *$ & $-3.67 * *$ & $21.92 * *$ & $58.93 * *$ & $5.95 * *$ \\
\hline & S.Em \pm & 0.70 & 0.70 & 0.70 & 0.39 & 0.39 & 0.39 \\
\hline & C.D. @ $5 \%$ & 1.99 & 1.99 & 1.99 & 1.12 & 1.12 & 1.12 \\
\hline & C.D.@1\% & 2.67 & 2.67 & 2.67 & 1.50 & 1.50 & 1.50 \\
\hline
\end{tabular}

*and ** indicate significance of values at $\mathrm{p}=0.05$ and 0.01 , respectively. BP- Heterosis over better parent, BTPHeterosis over the best parent, $\mathrm{CC}$ - Heterosis over the commercial check, $\mathrm{KC}$-Kolkotta collection, $\mathrm{AB}=\mathrm{Arka}$ Bahar, $\mathrm{PN}=$ Pusa Naveen, S-Samrat 
Table.4 Heterosis (\%) over better parent, the best parent and the commercial check for days to first harvest and days to last harvest in bottle gourd

\begin{tabular}{|c|c|c|c|c|c|c|c|}
\hline \multirow{2}{*}{$\begin{array}{l}\text { Sl. } \\
\text { No. }\end{array}$} & \multirow[t]{2}{*}{ Cross } & \multicolumn{3}{|c|}{ Days to first harvest } & \multicolumn{3}{|c|}{ Days to last harvest } \\
\hline & & BP & BTP & $\mathrm{CC}$ & BP & BTP & $\mathrm{CC}$ \\
\hline 1 & Bot.G-2 X AB & $9.16 * *$ & $20.17 * *$ & 2.14 & 6.42 & -2.11 & $6.91 *$ \\
\hline 2 & Bot.G-2 X PN & $6.67 * *$ & $21.01 * *$ & 2.86 & -1.34 & $-6.75^{*}$ & 1.84 \\
\hline 3 & Bot.G-2 X S & 0.00 & $16.81 * *$ & -0.71 & -1.83 & $-9.71 * *$ & -1.38 \\
\hline 4 & Bot.G-2-1 X AB & $9.16 * *$ & $20.17 * *$ & 2.14 & 0.88 & -2.95 & 5.99 \\
\hline 5 & Bot.G-2-1 X PN & -1.48 & $11.77 * *$ & $-5.00 *$ & $-10.96 * *$ & $-14.35 * *$ & -6.45 \\
\hline 6 & Bot.G-2-1 X S & 0.00 & $16.81 * *$ & -0.71 & $-7.02 *$ & $-10.55 * *$ & -2.30 \\
\hline 7 & Bot.G-4 X AB & $15.00 * *$ & $15.97 * *$ & -1.43 & -1.86 & $-10.97 * *$ & -2.76 \\
\hline 8 & Bot.G-4 X PN & $16.67 * *$ & $17.65 * *$ & 0.00 & -4.91 & $-10.13 * *$ & -1.84 \\
\hline 9 & Bot.G-4 X S & $24.17 * *$ & $25.21 * *$ & $6.43 * *$ & 4.19 & -5.49 & 3.23 \\
\hline 10 & Bot.G-4-1 X AB & $6.11 * *$ & $16.81 * *$ & -0.71 & -5.63 & $-8.02 * *$ & 0.46 \\
\hline 11 & Bot.G-4-1 X PN & 2.22 & $15.97 * *$ & -1.43 & $-9.09 * *$ & $-11.39 * *$ & -3.23 \\
\hline 12 & Bot.G-4-1 X S & $7.35 * *$ & $22.69 * *$ & $4.29 *$ & 0.87 & -1.69 & $7.37 *$ \\
\hline 13 & Bot.G-6 X AB & $7.63 * *$ & $18.49 * *$ & 0.71 & $-6.75^{*}$ & $-6.75 *$ & 1.84 \\
\hline 14 & Bot.G-6 X PN & $4.44 *$ & $18.49 * *$ & 0.71 & $-7.59 *$ & $-7.60 * *$ & 0.92 \\
\hline 15 & Bot.G-6 X S & 3.60 & $21.01 * *$ & 2.86 & -2.11 & -2.11 & $6.91 *$ \\
\hline 16 & Bot.G-6-1 X AB & $7.63 * *$ & $18.49 * *$ & 0.71 & -3.51 & $-7.17 *$ & 1.38 \\
\hline 17 & Bot.G-6-1 X PN & $6.67 * *$ & $21.01 * *$ & 2.86 & -0.88 & -4.64 & 4.15 \\
\hline 18 & Bot.G-6-1 X S & $-5.04 *$ & $10.92 * *$ & $-5.71 *$ & $-9.65^{* *}$ & $-13.08 * *$ & -5.07 \\
\hline 19 & Bot.G-6-2 X AB & $9.16 * *$ & $20.17 * *$ & 2.14 & -3.45 & -5.49 & 3.23 \\
\hline 20 & Bot.G-6-2 X PN & 3.70 & $17.65^{* *}$ & 0.00 & -2.59 & -4.64 & 4.15 \\
\hline 21 & Bot.G-6-2 X S & 2.16 & $19.33 * *$ & 1.43 & $-7.76^{* *}$ & $-9.71 * *$ & -1.38 \\
\hline 22 & Bot.G-7 X AB & $7.63 * *$ & $18.49 * *$ & 0.71 & $-9.40 * *$ & $-10.55 * *$ & -2.30 \\
\hline 23 & Bot.G-7 X PN & 1.48 & $15.13 * *$ & -2.14 & -6.41 & $-7.60 * *$ & 0.92 \\
\hline 24 & Bot.G-7 X S & 0.72 & $17.65 * *$ & 0.00 & $-9.40 * *$ & $-10.55 * *$ & -2.30 \\
\hline 25 & Bot.G-7-1 X AB & $12.98 * *$ & $24.37 * *$ & $5.71 *$ & $7.25^{*}$ & -6.33 & 2.30 \\
\hline 26 & Bot.G-7-1 X PN & 3.70 & $17.65 * *$ & 0.00 & -1.34 & $-6.75^{*}$ & 1.84 \\
\hline 27 & Bot.G-7-1 X S & 2.88 & $20.17 * *$ & 2.14 & $12.08 * *$ & -2.11 & $6.91 *$ \\
\hline 28 & $\mathrm{KC} X \mathrm{AB}$ & $4.58 *$ & $15.13 * *$ & -2.14 & $-1.92 *$ & $-13.92 * *$ & -5.99 \\
\hline 29 & $\mathrm{KC} X \mathrm{PN}$ & -3.70 & $9.24 * *$ & $-7.14 * *$ & $-7.14 *$ & $-12.24 * *$ & -4.15 \\
\hline 30 & KC X S & 2.22 & $15.97 * *$ & -1.43 & 0.00 & $-12.24 * *$ & -4.15 \\
\hline & S.Em \pm & 1.55 & 1.55 & 1.55 & 2.32 & 2.32 & 2.32 \\
\hline & C.D. @ $5 \%$ & 4.44 & 4.44 & 4.44 & 6.62 & 6.62 & 6.62 \\
\hline & C.D.@1\% & 5.93 & 5.93 & 5.93 & 8.85 & 8.85 & 8.85 \\
\hline
\end{tabular}

*and ** indicate significance of values at $\mathrm{p}=0.05$ and 0.01 , respectively. BP- Heterosis over better parent, BTPHeterosis over the best parent, $\mathrm{CC}$ - Heterosis over the commercial check, KC-Kolkotta collection, $\mathrm{AB}=\mathrm{Arka}$ Bahar, $\mathrm{PN}=$ Pusa Naveen, S-Samrat 
Table.5 Heterosis (\%) over better parent, the best parent and the commercial check for sex ratio and fruit yield per vine in bottle gourd

\begin{tabular}{|c|c|c|c|c|c|c|c|}
\hline \multirow{2}{*}{$\begin{array}{l}\text { Sl. } \\
\text { No. }\end{array}$} & \multirow[t]{2}{*}{ Cross } & \multicolumn{3}{|c|}{ Sex ratio } & \multicolumn{3}{|c|}{ Fruit yield per vine } \\
\hline & & BP & BTP & $\mathrm{CC}$ & BP & BTP & $\mathrm{CC}$ \\
\hline 1 & Bot.G-2 X AB & - & $9.04 * *$ & $-1.14 * *$ & $21.09 * *$ & $11.14 * *$ & $16.99 * *$ \\
\hline 2 & Bot.G-2 X PN & $-9.55 * *$ & $13.49 * *$ & $2.90 * *$ & $5.45 * *$ & $-3.21 * *$ & $1.88 * *$ \\
\hline 3 & Bot.G-2 X S & - & $15.97 * *$ & $5.15 * *$ & $14.79 * *$ & $5.36 * *$ & $10.90 * *$ \\
\hline 4 & Bot.G-2-1 X AB & - & $4.33 * *$ & $-5.41 * *$ & $43.18 * *$ & $12.50 * *$ & $18.42 * *$ \\
\hline 5 & Bot.G-2-1 X PN & $2.55^{* *}$ & $22.40 * *$ & $10.98 * *$ & $25.00 * *$ & $-1.79 * *$ & $3.38 * *$ \\
\hline 6 & Bot.G-2-1 X S & $-7.73 * *$ & $10.14 * *$ & -0.14 & $36.82 * *$ & $7.50 * *$ & $13.16 * *$ \\
\hline 7 & Bot.G-4 X AB & $-2.28 * *$ & $16.79 * *$ & $5.89 * *$ & $37.03 * *$ & $8.64 * *$ & $14.36 * *$ \\
\hline 8 & Bot.G-4 X PN & - & $-5.87 * *$ & - & $44.14 * *$ & $14.29 * *$ & $20.30 * *$ \\
\hline 9 & Bot.G-4 X S & $-9.32 * *$ & $8.38 * *$ & $-1.74 * *$ & $29.37 * *$ & $2.57 * *$ & $7.97 * *$ \\
\hline 10 & Bot.G-4-1 X AB & - & $4.27 * *$ & $-5.46 * *$ & $16.85 * *$ & $13.93 * *$ & $19.92 * *$ \\
\hline 11 & Bot.G-4-1 X PN & - & -0.22 & $-9.53 * *$ & $9.89 * *$ & $7.14 * *$ & $12.78 * *$ \\
\hline 12 & Bot.G-4-1 X S & - & $8.16 * *$ & $-1.93 * *$ & $2.56^{* *}$ & 0.00 & $5.26 * *$ \\
\hline 13 & Bot.G-6 X AB & $6.87 * *$ & $35.21 * *$ & $22.59 * *$ & $13.12 * *$ & $-5.79 * *$ & $-0.83 * *$ \\
\hline 14 & Bot.G-6 X PN & - & $10.89 * *$ & 0.54 & $35.08 * *$ & $12.50 * *$ & $18.42 * *$ \\
\hline 15 & Bot.G-6 X S & - & $-2.35 * *$ & - & $38.08 * *$ & $15.00 * *$ & $21.05 * *$ \\
\hline 16 & Bot.G-6-1 X AB & $-2.75^{* *}$ & $5.37 * *$ & $-4.47 * *$ & $12.56 * *$ & $9.43 * *$ & $15.19 * *$ \\
\hline 17 & Bot.G-6-1 X PN & $6.63 * *$ & $15.53 * *$ & $4.75 * *$ & $-2.28 * *$ & $-5.00 * *$ & 0.00 \\
\hline 18 & Bot.G-6-1 X S & $-4.43 * *$ & $3.55 * *$ & $-6.12 * *$ & $20.13 * *$ & $16.79 * *$ & $22.93 * *$ \\
\hline 19 & Bot.G-6-2 X AB & -0.03 & -0.03 & $-9.36 * *$ & $24.51 * *$ & $14.29 * *$ & $20.30 * *$ \\
\hline 20 & Bot.G-6-2 X PN & $16.28 * *$ & $16.29 * *$ & $5.43 * *$ & $11.67 * *$ & $2.50 * *$ & $7.89 * *$ \\
\hline 21 & Bot.G-6-2 X S & $18.92 * *$ & $18.92 * *$ & $7.82 * *$ & $0.39 *$ & $-7.86 * *$ & $-3.01 * *$ \\
\hline 22 & Bot.G-7 X AB & - & $-7.72 * *$ & - & $37.64 * *$ & $8.93 * *$ & $14.66 * *$ \\
\hline 23 & Bot.G-7 X PN & - & $11.33 * *$ & $0.94 *$ & $34.48 * *$ & $6.43 * *$ & $12.03 * *$ \\
\hline 24 & Bot.G-7 X S & - & $5.99 * *$ & $-3.90 * *$ & $37.55 * *$ & $8.86 * *$ & $14.59 * *$ \\
\hline 25 & Bot.G-7-1 X AB & $16.96 * *$ & $29.18 * *$ & $17.13 * *$ & $-6.85 * *$ & - & -10.00 \\
\hline 26 & Bot.G-7-1 X PN & $6.11 * *$ & $17.20 * *$ & $6.26 * *$ & $11.52 * *$ & $2.36 * *$ & $7.74 * *$ \\
\hline 27 & Bot.G-7-1 X S & $3.69 * *$ & $14.53 * *$ & $3.84 * *$ & $13.00 * *$ & $3.71 * *$ & $9.17 * *$ \\
\hline 28 & KC X AB & - & $-12.74 * *$ & - & $14.79 * *$ & $5.36 * *$ & $10.90 * *$ \\
\hline 29 & KC X PN & $3.80 * *$ & $19.20 * *$ & $8.08 * *$ & $14.79 * *$ & $5.36 * *$ & $10.90 * *$ \\
\hline 30 & $\mathrm{KC} \times \mathrm{S}$ & - & $-2.67 * *$ & - & $27.24 * *$ & $16.79 * *$ & $22.93 * *$ \\
\hline & S.Em \pm & 0.27 & 0.27 & 0.27 & 0.13 & 0.13 & 0.13 \\
\hline & C.D. @ $5 \%$ & 0.78 & 0.78 & 0.78 & 0.37 & 0.37 & 0.37 \\
\hline & C.D. @ 1\% & 1.04 & 1.04 & 1.04 & 0.49 & 0.49 & 0.49 \\
\hline
\end{tabular}

*and $* *$ indicate significance of values at $\mathrm{p}=0.05$ and 0.01 , respectively. BP- Heterosis over better parent, BTPHeterosis over the best parent, $\mathrm{CC}$ - Heterosis over the commercial check, KC-Kolkotta collection, $\mathrm{AB}=\mathrm{Arka}$ Bahar, $\mathrm{PN}=$ Pusa Nuveen, S-Samrat 
For sex ratio, out of 30 crosses, 20 crosses over better parent, five crosses over the best parent and 15 crosses over the commercial check exhibited significant negative heterosis. The maximum and significantly negative heterosis over better parent $(-33.87 \%)$ observed in the cross Bot.G-7 X Arka Bahar and maximum negative heterosis over the best parent $(-12.74 \%)$ and commercial check ($20.88 \%$ ) was observed in the cross Kolkotta Collection X Arka Bahar, which is low as compared to -51.51 per cent as reported by Sreevani (2004) in bottle gourd.

For fruit yield per vine, 27 crosses over better parent, 23 crosses over the best parent and 26 crosses over the commercial check showed positive and significant heterosis. The maximum and positively significant heterosis over better parent exhibited by the cross Bot.G-4 X Pusa Naveen (44.14\%) followed by Bot.G-2-1 X Arka Bahar (43.18\%) and is more or less similar as compared to 46.34 per cent as reported by Ray et al., (2015). The crosses Bot.G-6-1 X Samrat and Kolkotta Collection X Samrat (16.79\%) followed by Bot.G-6 X Samrat (15.00\%) showed significant heterosis over the best parent and is little more as compared to 13.80 per cent as reported by Kumar et al., (1999). The crosses Bot.G-6-1 X Samrat and Kolkotta Collection X Samrat (22.93\%) followed by Bot.G-6 X Samrat $(21.05 \%)$ showed significant heterosis over the commercial check in order of merit and are similar as compared to 21.32 per cent reported by Adarsh et al., (2017) in bottle gourd.

From over all study, the results were summarized that the crosses Bot.G-6-1 X Samrat and Kolkotta Collection X Samrat were said to be the best crosses recording $22.93 \%$ of commercial heterosis with fruit yield of $8.18 \mathrm{~kg} / \mathrm{vine}$. These crosses also exhibited significant and high standard heterosis in the desirable direction for number of branches and sex ratio. The second best cross Bot.G-6 X Samrat was selected based on fruit yield per vine which had total yield of $8.05 \mathrm{~kg} / \mathrm{vine}$ with 21.05 per cent standard heterosis and this cross exhibited significant and standard heterosis in the desirable direction for nodes to first female flowering, days to last harvest and sex ratio. The next best crosses were Bot.G-4 X PN and Bot.G-62 X AB exhibited 20.30 per cent standard heterosis for fruit yield per vine which had yielding ability of $8.00 \mathrm{~kg} / \mathrm{vine}$. This hybrid exhibited significant and standard heterosis in the desirable direction for number of nodes upto first female flowering and sex ratio. These crosses can be further assessed for its yield stability to confirm its potentiality and adaptability to different agro-climatic conditions before its commercial exploitation.

\section{References}

Adarsh, A., Kumar, R., Kumar, A., Singh, H. K. and Neetunand, 2017, Estimation of gene action and heterosis in bottle gourd (Lagenaria siceraria Mol. Standl.). Environment and Ecology, 35(2A): 936944.

Dubey, S. K. and Maurya, I. B., 2003, Studies on heterosis and combining ability studies in bottle gourd [Lagenaria siceraria (Mol.) Standl.]. Indian $J$. Genet., 63(2): 148-152.

Gayakawad, 2014, Heterosis and combining ability studies in bottle gourd [Lagenaria siceraria (Mol.) Standl.]. $M$. Sc. (Hort.) Thesis, Univ. Hort. Sci., Bagalkot.

Ghuge, M. B., Syamal, M. M. and Karcho, S., 2016, Heterosis in bottle gourd [Lagenaria siceraria (Mol.) Standl.]. Indian J. Agric. Res., 50(5): 466-470.

Naliyadhara, M. V., Dhaduk, L. K., Barad, A. V., Purohit, V. L. and Vachhani, J. H., 2007, Heterosis for fruit yield and its components in sponge gourd (Luffa 
cylindrica (Roem.) L.). Nat. J. Plant. Improv., 9(2): 132-135.

Pandey, S. K., Shrivastava, B. P., Shrivastava, S. B. L. and Shrivastava, J. P., 2004, Heterosis and inbreeding depression for fruit characters in bottle gourd [Lagenaria siceraria (Mol.) Standl.]. Indian J. Hort., 61(120): 146-149.

Ray, P. K., Yadav, G. C., Baranwal, D. K. and Singh, H. K., 2015, Genetic estimates and gene action for obtaining promising heterotic hybrids in bottle gourd [Lagenaria siceraria (Mol.) Standl.]. The Bioscan, 10(2): 801-806.

Sharma, D. R., Choudhary, M. R., Jakhar, M. L. and Dadheech S., 2012, Heterosis in bottle gourd [Lagenaria siceraria
(Mol.) Standl.]. Int. J. Life Sci., 1(3): 212-216.

Singh, S. K., Singh, R. K., Shashank, S. S. and Upadhyay, A. K., 2012, Studies on genetic causes of heterosis in bottle gourd [Lagenaria siceraria (Mol.) Standl.] near Gangatic region of Varanasi. Asian J. Hort., 7(2): 303-306.

Sreevani, P. G., 2004, Studies on exploitation of heterosis in bottle gourd [Lagenaria siceraria (Mol.) Standl.]. Madras Agric. J., 91(4-6): 311-313.

Yadav, Y. C. and Kumar, S., 2012, Assessment of standard heterosis for crop improvement in bottle gourd [Lagenaria siceraria (Mol.) Standl.]. Int. J. Plant Sci., 7(1): 181-184.

\section{How to cite this article:}

Raju K. Khot, Shashikanth Evoor, Vilas D. Gasti, Sumangala Koulagi and Dileepkumar A. Masuthi. 2018. Estimation of Heterosis in the Advanced Lines of Bottle Gourd [Lagenaria siceraria (Mol.) Standl.] for Growth, Earliness and Yield Parameters. Int.J.Curr.Microbiol.App.Sci. 7(09): 3375-3384. doi: https://doi.org/10.20546/ijcmas.2018.709.419 